\title{
Giant and Rapidly Growing Malignant Peripheral Nerve Sheath Tumor of the Head and Neck with Pulmonary Metastasis: Case Report and Review of the Literature
}

\author{
Yuandong Cao ( $\nabla$ caoyuandong888@126.com ) \\ The First Affiliated Hospital with Nanjing Medical University \\ Wei Tao \\ Nanjing Pukou Central Hospital \\ Ting Jia \\ Nanjing Pukou Central Hospital \\ Liang Liu \\ Nanjing Pukou Central Hospital \\ Chunyu Jiang \\ Nanjing Pukou Central Hospital
}

\section{Case Report}

Keywords: Malignant peripheral nerve sheath tumor, head and neck tumor, radiotherapy, antiangiogenic therapy

Posted Date: March 7th, 2022

DOI: https://doi.org/10.21203/rs.3.rs-1414544/v1

License: (c) (1) This work is licensed under a Creative Commons Attribution 4.0 International License.

Read Full License 


\section{Abstract}

Background $\mathbb{M}$ Malignant peripheral nerve sheath tumors (MPNSTs) are a subtype of malignant soft tissue sarcoma arising from peripheral nerve branches or sheaths of peripheral nerve fibers. Most cases are associated with bigger nerve stems, and are more commonly found in the proximal parts of the limbs and trunk, but rarely in the head and neck regions. However, reports on MPNSTs with pulmonary metastasis at the time of initial diagnosis are limited. Here, we present a case of MPNST that is located in the head and neck region with pulmonary metastasis and a literature review.

Case description区Here, we report a case of primary malignant peripheral nerve sheath tumor of the head and neck with pulmonary metastasis. The tumor was surgically removed and fused with autogenous bone and artificial bone. The histopathological diagnosis was MPNST. However, the tumor rapidly recurred one month after surgery, and the patient exhibited progressive decline in muscle strength with numbness and an inability to move independently, so the tumor was again surgically removed. Then, she underwent adjuvant radiotherapy, chemotherapy, and antiangiogenic therapy. After three months, the patient's tumor rapidly progressed into intracranial, neck, and lung lesions, and genetic tests were performed.

Conclusions: Giant MPNSTs of the head and neck with pulmonary metastasis are highly aggressive lesions that should primarily be treated in a surgical fashion. Adjuvant radiotherapy should be initiated as soon as the patient recovers from the postoperative wound, preferably within two weeks. Antiangiogenic drugs and radiotherapy may have synergistic effects.

\section{Background}

Malignant peripheral nerve sheath tumors (MPNSTs) are rare malignant tumors with poorly understood etiology, accounting for $5 \%-10 \%$ of all soft tissue sarcomas $[1,2]$. Theoretically, MPNSTs occur in peripheral nerves of the body, and because these tumors often arise from major nerve branches, the disease commonly occurs in the trunk and proximal parts of the limbs, such as the brachial plexus, thigh, sciatic nerve, buttocks and paravertebral region $[3,4]$. For patients with MPNSTs, despite surgery, adjuvant radiotherapy and chemotherapy, the overall prognosis is poor [5]. Of all soft-tissue sarcomas, MPNSTs have the highest mortality rate [6].

\section{Case Presentation}

A 20-year-old female presented with rapidly enlarging swelling in the neck and head within a period of 3 months. She had no past medical history related to neurofibromatosis and only had symptoms local soreness. The initial magnetic resonance imaging (MRI) showed a left posterior occipital area and upper neck tumor of $6.7 \mathrm{~cm} \cdot 6.0 \mathrm{~cm} \cdot 5.5 \mathrm{~cm}$ in size, with the cervical spinal cord was slightly compressed (Figs. 1A and B). Cervical vertebra computed tomography (CT) showed local osteolytic destruction of the left mass of the atlas (Fig. 1C). Chest CT showed bilateral, multiple, variably sized pulmonary nodules 
consistent with pulmonary metastasis, the largest of which measured $3.3 \mathrm{~cm} \cdot 2.5 \mathrm{~cm} \cdot 2.2 \mathrm{~cm}$ in the left inferior lobe of the lung (Fig. 1D).

Initially, the patient underwent surgical removal of the tumor at our hospital on January 5, 2021. During the operation, the tumor was found to be located on the left side of the C1-C2 spinal canal, invading the surrounding muscle tissue, tough in quality, with an incomplete capsule, with extremely rich blood supply, and partially protruding into the spinal canal. The tumor tissue was removed nearly completely under the microscope. Pathological examination revealed the typical appearance of MPNST with densely cellular areas alternating with less cellular areas (Fig. 2A-C). Most areas are less cellular areas, with background myxoid degeneration and chronic inflammatory cell infiltration. In some densely cellular areas, the cells showed marked atypia, mitosis, and necrosis, and the tumor tissue infiltrated the surrounding rhabdoid muscle. Immunohistochemical analysis indicated that the tissue stained positive for S-100, SOX-10, and $\mathrm{Ki}-67(10+)$, and negative for H3K27me3, CD34, and SMA in less cellular areas, while positive for Ki$67(80+)$, and negative for S-100, SOX-10, H3K27me3, CD34, and SMA in densely cellular areas.

About two weeks later, the patient felt mild numbness in both upper limbs, mainly in the left upper limbs, although limb muscle strength was normal, without paying attention to it. On February 16, 2021, the patient suddenly felt weakness in the limbs and could not move by herself, and was again admitted to our hospital. Cervical spine MRI showed the tumor recurrence, measuring $5.8 \mathrm{~cm} \cdot 5.5 \mathrm{~cm} \cdot 5.0 \mathrm{~cm}$ (Figs. 3A and B). Chest CT scan showed a left lung mass was that measured $3.5 \mathrm{~cm} \cdot 3.3 \mathrm{~cm} \cdot 2.8 \mathrm{~cm}$, slightly larger than that observed on December 28, 2020 (Fig. 3C).

Then, the patient underwent epidural tumor resection again on February 22, 2021. Postoperative pathology diagnosed the tumor as an MPNST. After the operation, the patient could get out of bed and move.

Three weeks after second surgery, the patient suddenly felt weakness again in the left limbs. On March 16, 2021, cervical spine MRI showed irregular space occupying lesions in the left cerebellopontine angle region and left cerebellar lower to neck, measuring $7.0 \mathrm{~cm} \cdot 4.8 \mathrm{~cm} \cdot 4.5 \mathrm{~cm}$, which was considered tumor recurrence with left internal jugular vein invasion (Figs. 4A and B). Chest CT examination showed that the new lesion measured $1.8 \mathrm{~cm} \cdot 1.5 \mathrm{~cm} \cdot 1.0 \mathrm{~cm}$ in the right lung (Fig. 4C). After multidisciplinary consultation and discussion, radiotherapy was our first choice. However, while waiting for radiotherapy, her symptoms were progressively aggravated, and she was by then unable to get out of bed. To prevent the rapid growth of the tumor, we had to first give epirubicin $50 \mathrm{mg} \mathrm{D} 1$ and D2 + cisplatin $40 \mathrm{mg}$ D1 and D2 and $20 \mathrm{mg}$ D3 for postoperative adjuvant chemotherapy once on March 19, 2021, and the tumor radiotherapy was initiated on March 19, 2021, with the doses of PGTV 52.0Gy/26f and PTV 46.8Gy/26f, and three times of cisplatin $40 \mathrm{mg}$ per week were administered during radiotherapy. There was no improvement in the patient's symptoms at 10 times of radiotherapy, and antivascular therapy with anlotinib once a day $(10 \mathrm{mg})$ from days $1-14$ of a 21-day cycle was added on April 02, 2021. One week after the addition of anlotinib, the patient's symptoms significantly improved, and she was then able to move independently. 
Subsequently, on May 25, 2021, the patient's head and neck MRI showed that the lesions were slightly reduced (Figs. 5A and B), while chest CT showed an increased rate of enlargement of pulmonary lesions (Fig. 5C). The therapeutic effect of the disease showed stable disease in the head and neck lesions, and progressive disease in chest lesions. Considering the severe digestive tract reaction of the patient after previous chemotherapy with cisplatin, temozolomide 200 mg IV D1-5 + epirubicin 50 mg IV D1-2 was given on May 22, 2021, and antivascular therapy with anlotinib was continued.

On June 25, 2021, the patient felt weakness in the limbs, and cervical spine MRI showed that the tumor in the head and neck were still reduced (Fig. 6A), with multiple intracranial metastases (Figs. 6B and C). Chest CT showed increased and enlarged double lung lesions (Fig. 6D). Comprehensive evaluation of the curative effect indicated progressive disease. Then, the patient underwent whole-genome testing, but no pathogenic variants were identified and died the following month.

\section{Discussion}

An MPNST is a relatively rare but highly malignant sarcoma of soft tissues [7]. It primarily occurs with a peak incidence between the ages of 30 and 60 years of life in the general population with equal frequency in males and females, although these tumors may occur at a much younger age [8]. The case reported here is a 20 -year-old girl, indicating that age predisposition is contributory factor of disease.

MPNSTs are associated with inherited abnormalities, such as the loss of neurofibromatosis 1 (NF1) gene protein. NF1 is an autosomal dominant disorder that represents the most common human cancer genetic susceptibility syndrome with an incidence of approximately 1 in 3,000 [9]. NF1 is characterized by multiple areas of cutaneous hyperpigmentation, axillary freckling, numerous neurofibromas, optic gliomas, bone dysplasia, iris hamartomas termed Lisch nodules, and a family history of NF1 in a firstdegree relative, and it is diagnosed in the presence of any two of these seven criteria [9]. Half of MPNSTs derive from nerves affected by NF1, and the other half occurs in the general population. Among the other half of MPNSTs that occur in the general population, approximately $40 \%$ are sporadic and the remaining $10 \%$ arise secondary to previous irradiation [10]. Inferior outcomes have been observed in radiotherapyinduced MPNSTs compared to sporadic or NF1-related MPNSTs [11, 12]. Our patient had no clinical manifestation of any of the features of NF1 mentioned above, nor did she have any history of radiotherapy. Therefore, her case was considered as sporadic.

The histopathological features of MPNSTs are nonspecific. In general, tumors are composed almost entirely of compact spindle cells with variable hyperchromatism and pleomorphism arranged in intersecting fascicles [13]. In our case, histopathological examination showed that most areas are less cellular areas, with background myxoid degeneration and chronic inflammatory cell infiltration. In some densely cellular areas, the cells showed marked atypia, mitosis, and necrosis, and tumor tissue infiltrated the surrounding rhabdoid muscles. However, due to the uncertainty of histological diagnoses, immunohistochemical is essential for the definitive diagnosis of MPNST. The immunohistochemical results of our patient showed that S-100, SOX-10, Ki-67(10+) were all expressed in less cellular areas, 
while expression of Ki-67(80+), but not S-100 and SOX-10, was observed in densely cellular areas. Studies have shown that the immunoreactivity for S100 and SOX-10 proteins indicates Schwann cell origin and support a diagnosis of cellular schwannoma [14-16]. However, the sensitivities of S100 and SOX10 expression for the diagnosis of MPNST were not high, which suggests downregulation of Schwannian markers in MPNST $[13,17,18]$.

MPNSTs involve a high rate of metastasis via the bloodstream, while with infrequent but possible lymphatic spread [19]. Furthermore, there is a high rate of recurrence, with up to $50 \%$ local recurrence and up to $33 \%$ metastases to the lungs and bone $[20,21]$. According to several previously published studies, the five-year survival of head and neck MPNST ranges from $16-52 \%$, with better prognosis associated with complete resection, size $<5 \mathrm{~cm}$, high histological differentiation, and low Ki-67 labelling index [2227]. Our patient was considered to have residual tumor, size $>5 \mathrm{~cm}$, lung metastasis at the initial diagnosis, and Ki- 67 was $>80 \%$, and the survival time was only about six months after various treatments, which was considered to be caused by the high degree of malignancy and rapid progression of the tumor.

As with most soft tissue sarcomas, extensive resection is the primary treatment for MPNST [28-30]. Due to the high incidence of local recurrence in MPNSTs after surgery, adjuvant radiotherapy usually plays an indispensable role in local disease control [31, 32]. However, its role in reducing the incidence or improving survival in patients with distant metastases remains controversial [33-36]. In the present case, postoperative radiotherapy was delivered because of the extension of the tumor to the left atlas, which could harbor microscopic residual disease. However, the rapid progression of the lesion occurred within a short period of time before radiotherapy, and the patient did not have time for radiation therapy and had to undergo a second operation. However, three weeks after the second operation, the patient showed rapid progress again. After a multidisciplinary discussion, the patient was given radiotherapy combined with cisplatin and epirubicin chemotherapy. At the beginning, the patient's symptoms were not significantly relieved, but the patient's symptoms were significantly improved after the addition of antiangiogenesis treatment with Anlotinib. After the end of radiotherapy, reexamination showed that the lesions in the patient's head and neck were smaller than before, while the lung lesions were advanced. However, the patient's clinical symptoms were significantly improved and she by then was capable of moving independently. Considering the severe digestive tract reaction of the patient with cisplatin, we tried to give epirubicin combined with temozolomide intravenous chemotherapy, but the efficacy was unsatisfactory.

Currently, chemotherapy is only used for inoperable or very large tumors ( $>5-8 \mathrm{~cm}$ in size), incompletely resected cases, or patients with metastatic or recurrent disease [37]. Fortunately, the fact that the patient remained asymptomatic locally after two months of completion of radiotherapy combined with anlotinib indicates a possible benefit of the adjuvant postoperative radiotherapy combined with anlotinib, which might provide an alternative to the treatment of MPNST. Anlotinib is a novel, orally administered tyrosine kinases inhibitor that inhibits vascular endothelial growth factor receptor (VEGFR) signaling by selectively targeting VEGFR-2, -3 and fibroblast growth factor receptor 1 to $4[38,39]$. Recent studies showed a 
median progression-free survival and overall survival of 5.6 and 12 months, respectively, in patients with advanced soft tissue sarcoma treated with anroitinib [40]. Here, we, for the first time, demonstrate the clinical application of anlotinib combined with radiotherapy in the treatment of MPNST.

\section{Conclusions}

MPNSTs are highly malignant soft tissue sarcomas with a high short-term mortality rate, especially in the setting of metastatic disease. Once diagnosed with an MPNST, complete surgical resection should be performed. Postoperative radiotherapy is recommended as soon as possible. Additionally, other adjuvant therapies such as chemotherapy and tyrosine kinase inhibitor could also be considered for MPNST treatment. Larger studies and longer follow-up time are needed to validate our findings.

\section{Abbreviations}

MPNSTs, Malignant peripheral nerve sheath tumors; MRI, magnetic resonance imaging; $\mathrm{CT}$, computed tomography; NF1, neurofibromatosis 1; VEGFR, vascular endothelial growth factor receptor.

\section{Declarations}

\section{Acknowledgements}

The authors would like to thank all of the participants in the study.

\section{Conflict of interest}

None.

\section{Authors' contributions}

Wei Tao and Ting Jia made substantial contributions to conception and design of this case report and drafted the manuscript. Wei Tao, Ting Jia and Chunyu Jiang produced the data. Liang Liu made contributions to images collection. Wei Tao, Ting Jia, Chunyu Jiang and Yuandong Cao were involved in the acquisition and interpretation of the data and in revising the article. All authors read and approved the final manuscript.

\section{Funding}

The research was supported by Science and Technology Development Foundation of Nanjing Medical University (NMUB2019268). The founders had no role in the design and development of the study, data collection, analysis, writing of the report or decision to submit the paper for publication. 


\section{Availability of data and materials}

Data sharing is not applicable to this article as no datasets were generated or analysed during the current study.

\section{Ethics approval and consent to participate}

Not applicable.

\section{Consent for publication}

Written informed consent was obtained from the patient for publication of this Case report and any accompanying images. A copy of the written consent is available for review by the Editor-in-Chief of this journal.

\section{Competing interests}

The author declare that they have no competing interest.

\section{References}

1. Ng VY, Scharschmidt TJ, Mayerson JL, et al. Incidence and survival in sarcoma in the United States: a focus on musculoskeletal lesions. Anticancer Res. 2013; 33 (6): 2597-604.

2. Yi JM, Moon EJ, Oh SJ, et al. Malignant Peripheral Nerve Sheath Tumor of the Breast in a Patient without Neurofibromatosis: A Case Report. J Breast Cancer. 2009; 12 (3): 223-6.

3. Wang J, Ou S, Guo Z, et al. Microsurgical management of giant malignant peripheral nerve sheath tumor of the scalp: Two case reports and a literature review. World J Surg Oncol 2013;11 (269):1-8.

4. Firdaus M, Gill AS, Mukarramah DA, et al. Malignant Peripheral Nerve Sheath Tumor of the Scalp: Two Rare Case Reports. Surg Neurol Int 2018; 9 (102): 1-5.

5. Fan Q, Yang J, Wang G. Clinical and molecular prognostic predictors of malignant peripheral nerve sheath tumor. Clin Transl Oncol 2014; 16: 191-9.

6. Kim DH, Murovic JA, Tiel RL, et al. A series of 397 peripheral neural sheath tumors: 30-year experience at Louisiana State University Health Sciences Center. J Neurosurg. 2005; 102 (2): 246-55.

7. Fuchs B, Spinner RJ, Rock MG. Malignant peripheral nerve sheath tumors: an update. J Surg Orthop Adv. 2005; 14 (4): 168-74.

8. Ducatman BS, Scheithauer BW, Piepgras DG, et al. Malignant peripheral nerve sheath tumors. A clinicopathologic study of 120 cases. Cancer 1986; 57 (10): 2006-21. 
9. Farid M, Demicco EG, Garcia R, et al. Malignant Peripheral Nerve SheathTumors. Oncologist. 2014; 19: 193-201.

10. Laskin WB, Silverman TA, Enzinger FM. Postradiation Soft Tissue Sarcomas. An Analysis of 53 Cases. Cancer. 1988; 62 (11): 2330-40.

11. La Femina J, Qin LX, Moraco NH, et al. Oncologic outcomes of sporadic, neurofibromatosisassociated, and radiation-induced malignant peripheral nerve sheath tumors. Ann Surg Oncol. 2013; 20: 66-72.

12. Gladdy RA, Qin LX, Moraco N, et al. Doradiation-associated soft tissue sarcomas have the same prognosis as sporadic soft tissue sarcomas? J Clin Oncol. 2010; 28: 2064-69.

13. Pekmezci M, Reuss DE, Hirbe AC, et al. Morphologic and immunohistochemical features of malignant peripheral nerve sheath tumors and cellular schwannomas. Modern Pathology. 2015; 28 (2): 187-200.

14. Grobmyer SR, Reith JD, Shahlaee A, et al. Malignant Peripheral Nerve Sheath Tumor: Molecular Pathogenesis and Current Management Considerations. J Surg Oncol 2008; 97 (4): 340-9.

15. Ge P, Fu S, Lu L, et al. Diffuse scalp malignant peripheral nerve sheath tumor with intracranial extension in a patient with neurofibromatosis type 1. J Clin Neurosci 2010;17 (11): 1443-4.

16. Owosho AA, Estilo CL, Huryn JM, et al. A Clinicopathologic Study of Head and Neck Malignant Peripheral Nerve Sheath Tumors. Head Neck Pathol 2018; 12 (2): 151-9.

17. Miller SJ, Rangwala F, Williams J, et al. Large-scale molecular comparison of human schwann cells to malignant peripheral nerve sheath tumor cell lines and tissues. Cancer Res 2006; 66: 2584-91.

18. Karamchandani JR, Nielsen TO, van de Rijn M, et al. Sox10 and S100 in the diagnosis of soft-tissue neoplasms. Appl Immunohistochem Mol Morphol 2012; 20: 445-50.

19. Bailet JW, Abemayor E, Andrews JC, et al. Malignant nerve sheath tumors of the head and neck: a combined experience from two university hospitals. Laryngoscope.1991; 101: 1044-9.

20. Ma C, Ow A, Shan $\mathrm{OH}$, et al. Malignant peripheral nerve sheath tumours in the head and neck region: retrospective analysis of clinicopathological features and treatment outcomes. Int J Oral Maxillofac Surg. 2014; 43: 924-32.

21. Das Gupta TK, Brasfield RD. Solitary malignant schwannoma. Ann Surg.1970; 171: 419-28.

22. Zou C, Smith KD, Liu J, et al. Clinical, pathological, and molecular variables predictive of malignant peripheral nerve sheath tumor outcome. Ann Surg 2009; 249: 1014-22.

23. Heslin MJ, Cordon-Cardo C, Lewis JJ, et al. Ki-67 detected by MIB-1 predicts distant metastasis and tumor mortality in primary, high grade extremity soft tissue sarcoma. Cancer 1998; 83: 490-7.

24. Hruban RH, Shiu MH, Senie RT, et al. Malignant peripheral nerve sheath tumors of the buttock and lower extremity. A study of 43 cases. Cancer 1990; 66: 1253-65.

25. Vege DS, Chinoy RF, Ganesh B, et al. Malignant peripheral nerve sheath tumors of the head and neck: a clinicopathological study. J Surg Oncol 1994; 55: 100-3. 
26. Kourea HP, Bilsky MH, Leung DH, et al. Subdiaphragmatic and intrathoracic paraspinal malignant peripheral nerve sheath tumors: a clinicopathologic study of 25 patients and 26 tumors. Cancer 1998; 82: 2191-203.

27. Levine EA, Holzmayer T, Bacus $S$, et al. Evaluation of newer prognostic markers for adult soft tissue sarcomas. J Clin Oncol 1997;15: 3249-57.

28. Chao AH, Mayerson JL, Chandawarkar R, et al. Surgical management of soft tissue sarcomas: Extremity sarcomas. J Surg Oncol. 2014; 111 (5): 540-5.

29. Barker Jr JL, Paulino AC, Feeney S, et al. Locoregional treatment for adult soft tissue sarcomas of the head and neck: an institutional review. Cancer J 2003; 9 (1): 49-57.

30. Penel N, Van Haverbeke C, Lartigau E, et al. Head and neck soft tissue sarcomas of adult: prognostic value of surgery in multimodal therapeutic approach. Oral Oncol 2004; 40 (9): 890-7.

31. Garg A, Gupta V, Gaikwad SB, et al. Scalp malignant peripheral nerve sheath tumor (MPNST) with bony involvement and new bone formation: Case report. Clin Neurol Neurosurg 2004;106 (4): 340-4.

32. Kar M, Deo SV, Shukla NK, et al. Malignant peripheral nerve sheath tumors (MPNST)Clinicopathological study and treatment outcome of twenty-four cases. World J Surg Oncol 2006; 22 (8): $45-55$.

33. Yang JC, Chang AE, Baker AR, et al.Randomized prospective study of the benefit of adjuvant radiation therapy in the tatment of soft tissue sarcomas of the extremity. J Clin Oncol 1998; 16 (1):197-203.

34. Greager JA, Reichard KW, Campana JP, et al. Malignant schwannoma of the head and neck. Am J Surg 1992; 163: 440-2.

35. Hoffmann DF, Everts EC, Smith JD, et al. Malignant nerve sheath tumors of the head and neck. Otolaryngol Head Neck Surg 1988; 99: 309-14.

36. Vraa S, Keller J, Nielsen OS, et al. Prognostic factors in soft tissue sarcomas: the Aarhus experience. Eur J Cancer 1998; 34: 1876-82.

37. Moretti VM, Crawford EA, Staddon AP, et al. Early outcomes for malignant peripheral nerve sheath tumor treated with chemotherapy. Am J Clin Oncol 2011; 34: 417-21.

38. Chen XZ. Anlotinib for refractory advanced non-small cell lung Cancer in China. JAMA Oncol. 2019; 5: $116-7$.

39. Sun Y, Niu W, Du F, et al. Safety, pharmacokinetics, and antitumor properties of anlotinib, an oral multi-target tyrosine kinase inhibitor, in patients with advanced refractory solid tumors. Journal of Hematology \& Oncology 2016; 9(1): 105.

40. Chi Y, Fang ZW, Hong XN, et al. Safety and Efficacy of Anlotinib, a Multikinase Angiogenesis Inhibitor, in Patients with Refractory Metastatic Soft Tissue Sarcoma. Clin Cancer Res. 2018; 24 (21): 5233-8.

\section{Figures}




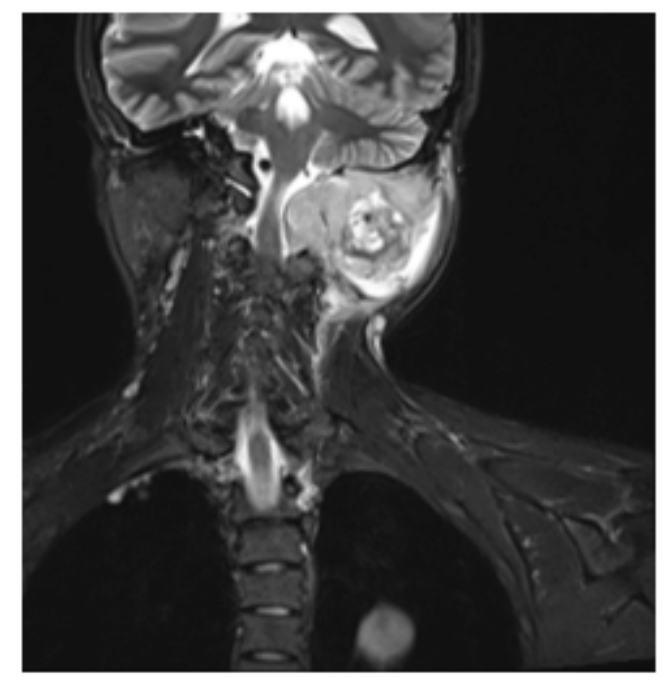

A

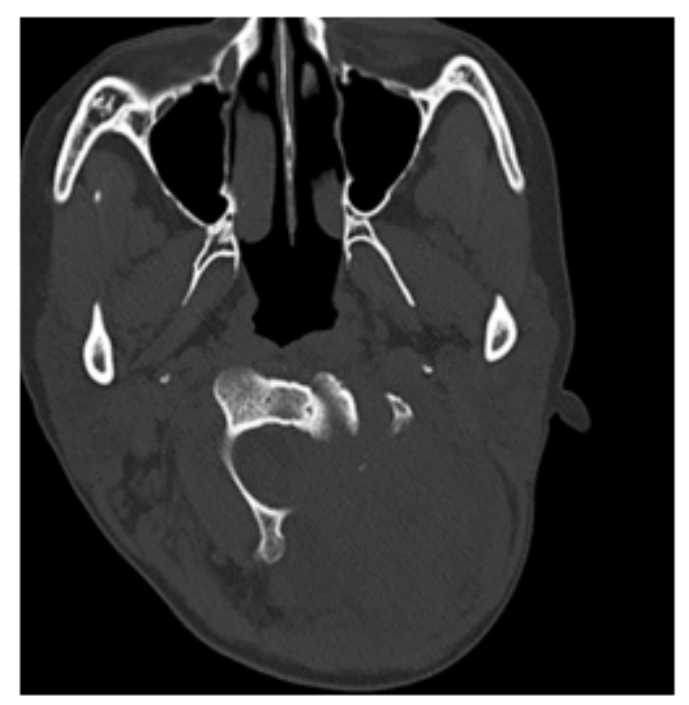

$\mathrm{C}$

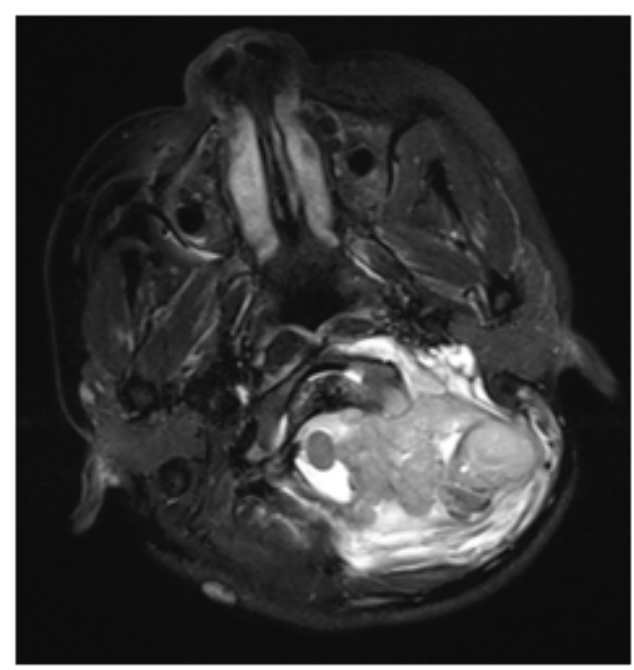

B

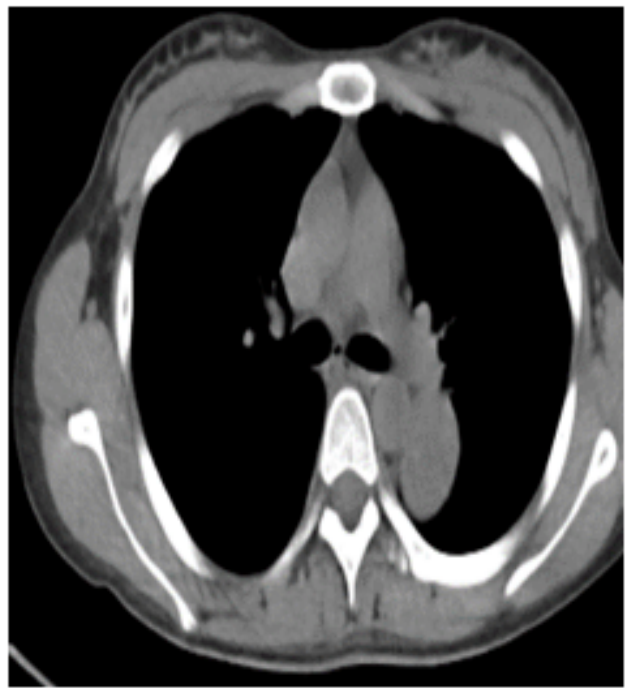

D

\section{Figure 1}

Imaging before the first surgery: Magnetic resonance imaging ( $A$ and $B$ ) showing a large left posterior occipital area and upper neck tumor with cervical spinal cord slightly compressed. Computed tomography scans (C) revealed local osteolytic destruction of the left mass of the atlas. Computed tomography scans (D) showing tumor focus on the left lung. 


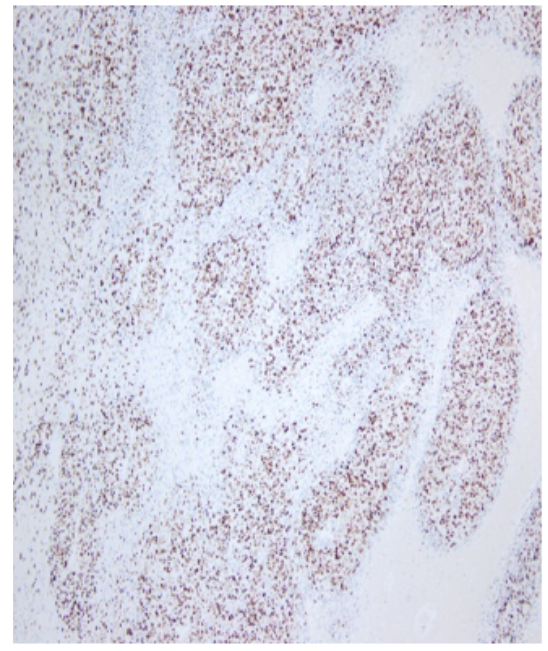

A

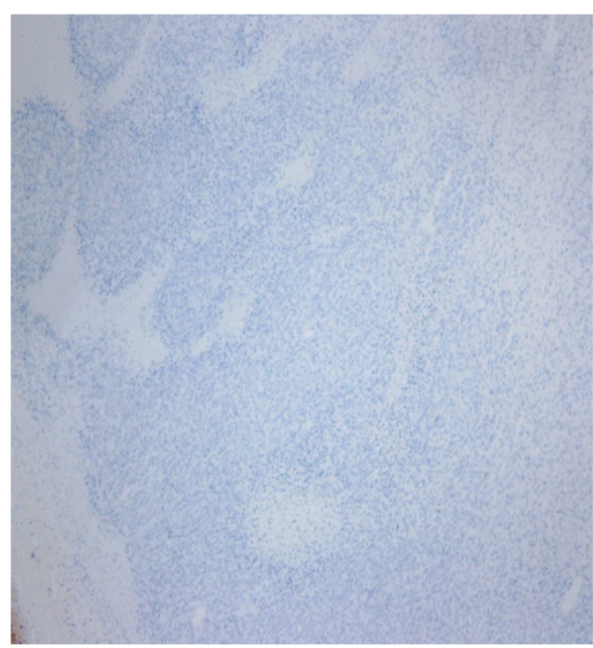

B

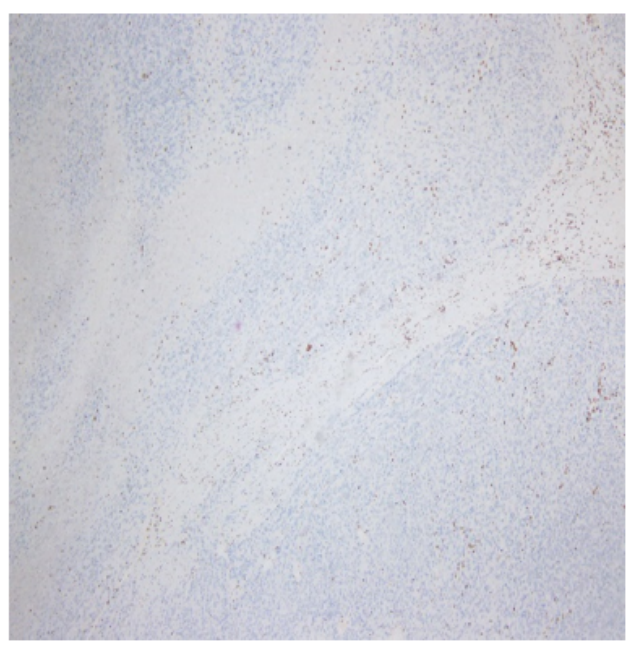

$\mathrm{C}$

\section{Figure 2}

Densely cellular areas showing (A) positive staining for Ki-67(80+), (B) negative staining for S-100, and (C) negative staining for H3K27me3.

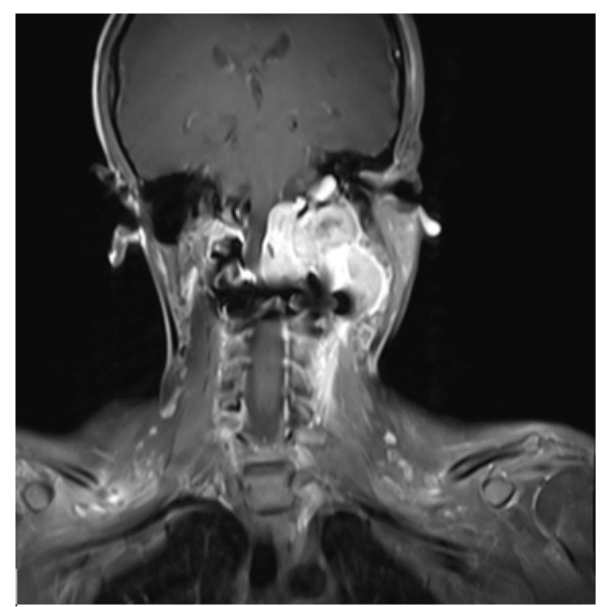

A

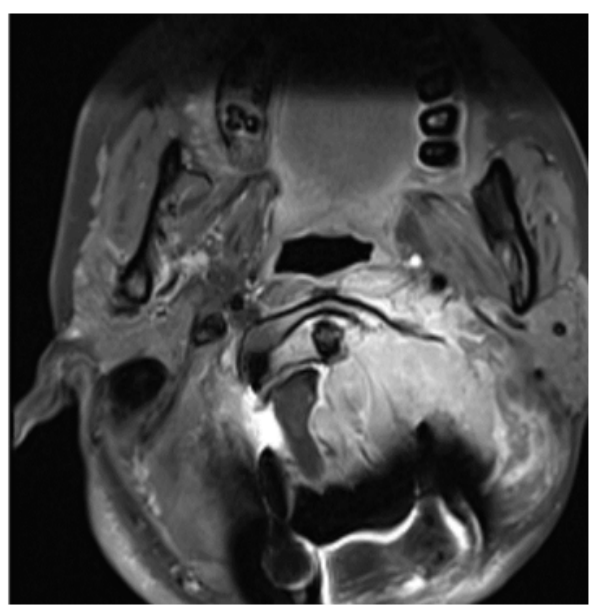

B

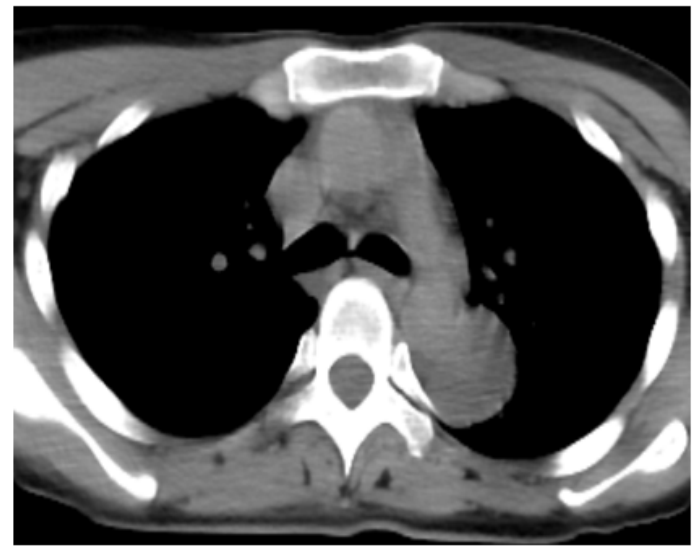

$\mathrm{C}$

Figure 3

Imaging before the second surgery: Magnetic resonance imaging ( $A$ and $B$ ) showing tumor recurrence in the head and neck, and the cervical spinal cord was slightly compressed. Computed tomography scans (C) revealed that the tumor in the left lung was slightly larger than prior evaluation. 


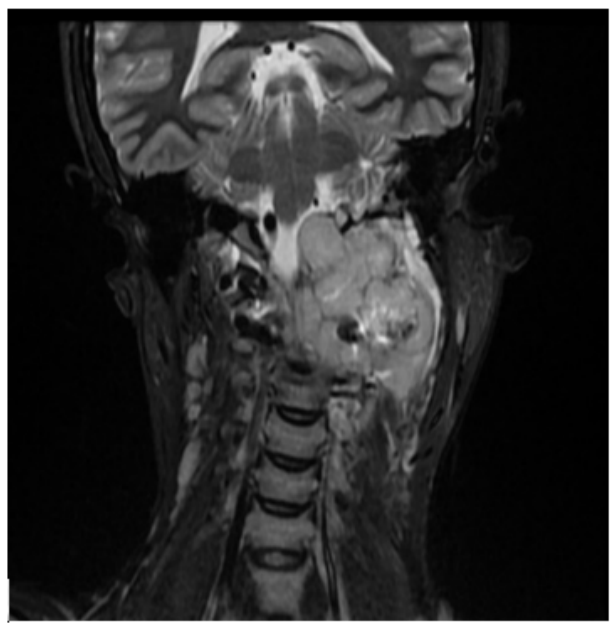

A

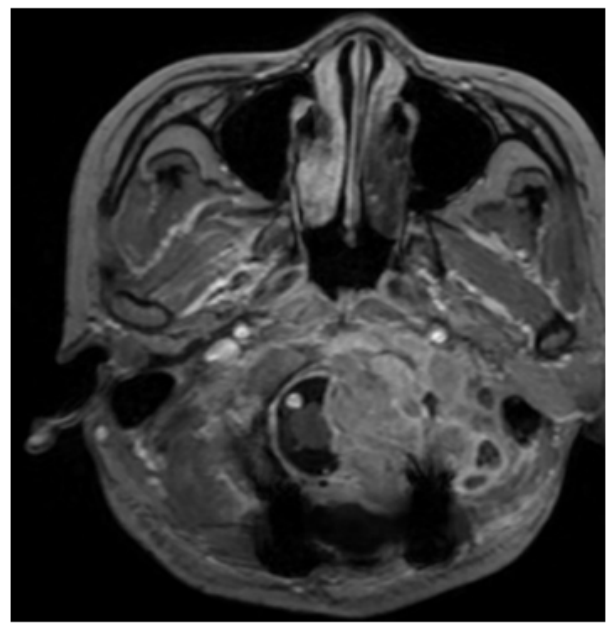

B

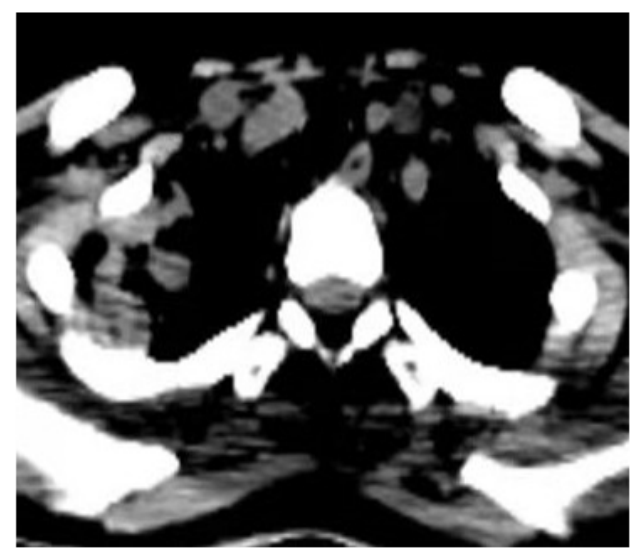

$\mathrm{C}$

\section{Figure 4}

Imaging before radiotherapy: Magnetic resonance imaging ( $A$ and $B$ ) showing tumor recurrence in the head and neck. Computed tomography scans (C) revealed a new rapidly growing tumor in the right lung.

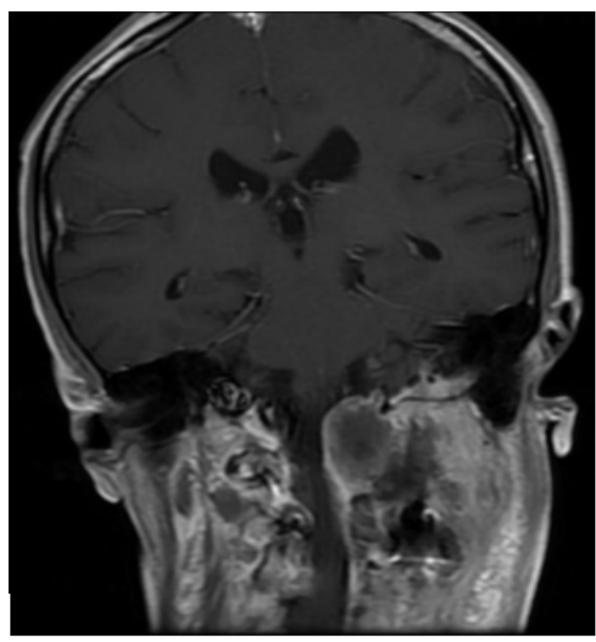

A

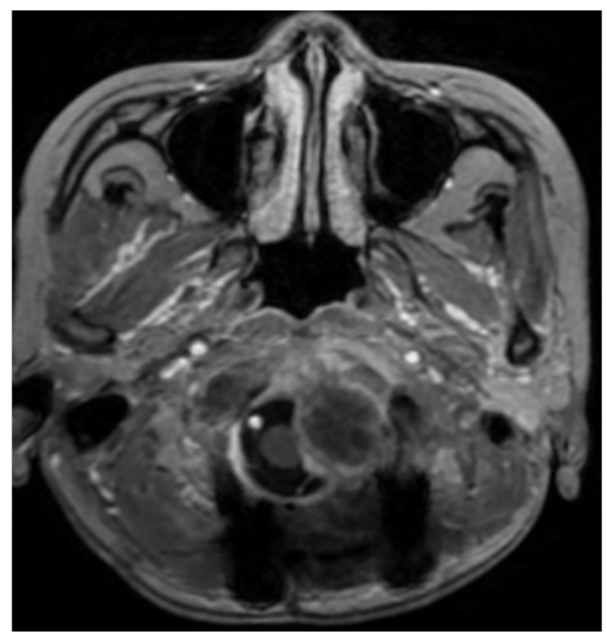

B

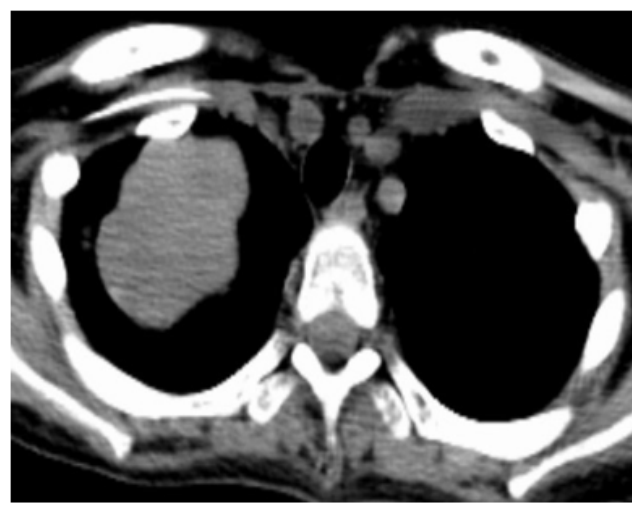

$\mathrm{C}$

\section{Figure 5}

Imaging one month after radiotherapy: Magnetic resonance imaging ( $A$ and $B$ ) showing the head and neck tumor had reduced in size due to liquefied necrosis. Computed tomography scans (C) revealed increased enlargement of right lung lesions compared to earlier evaluation. 


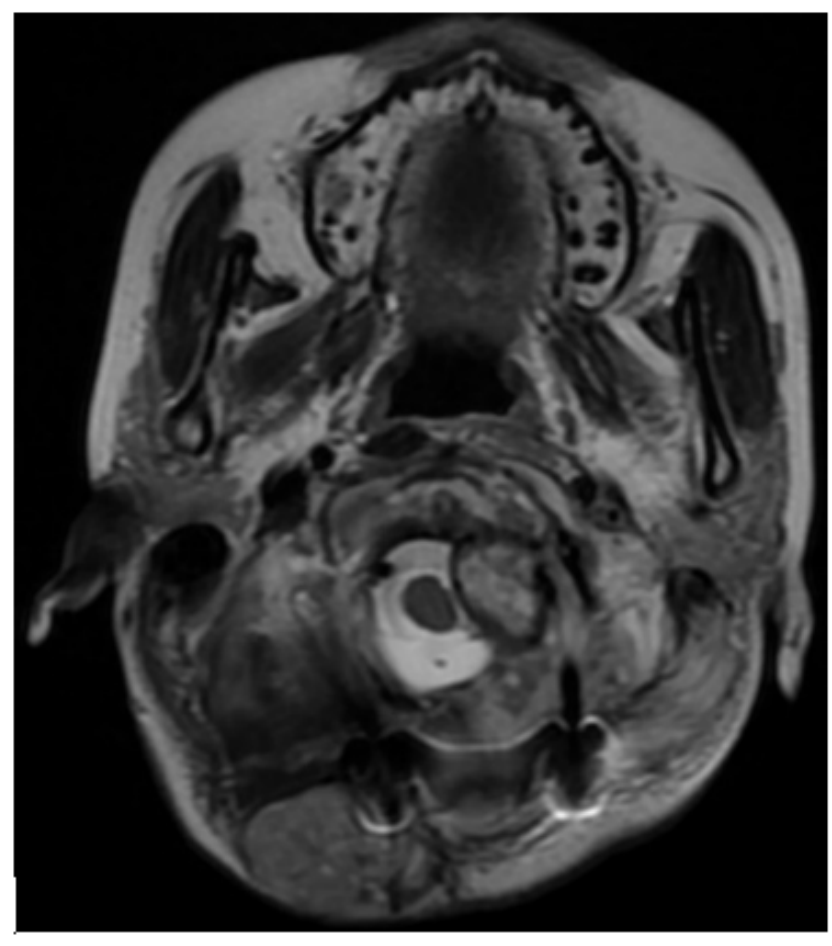

A

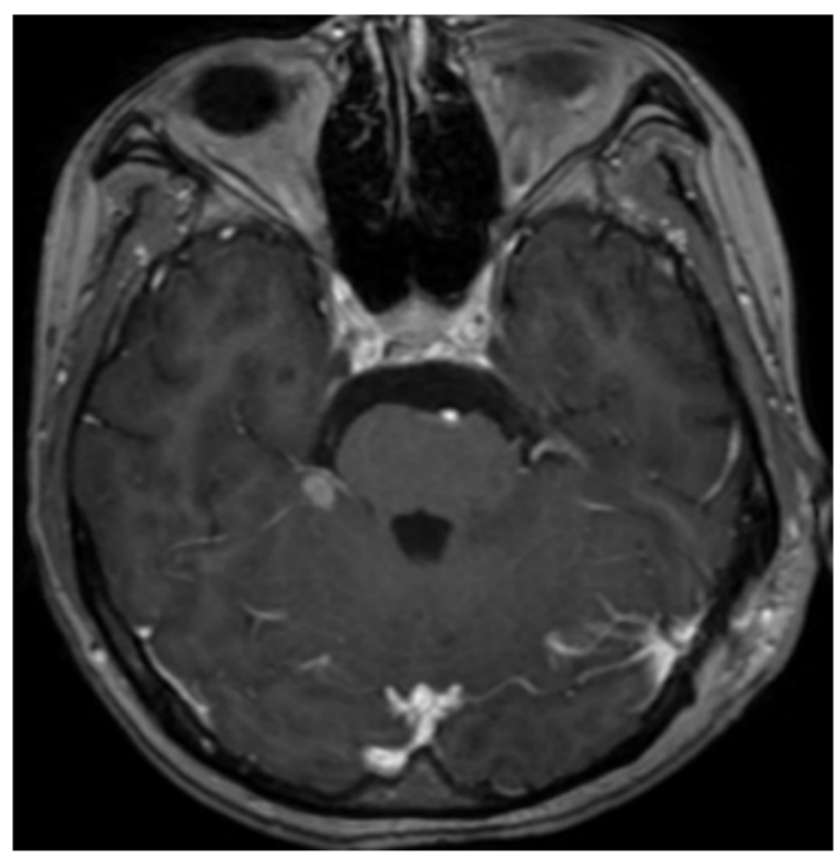

C

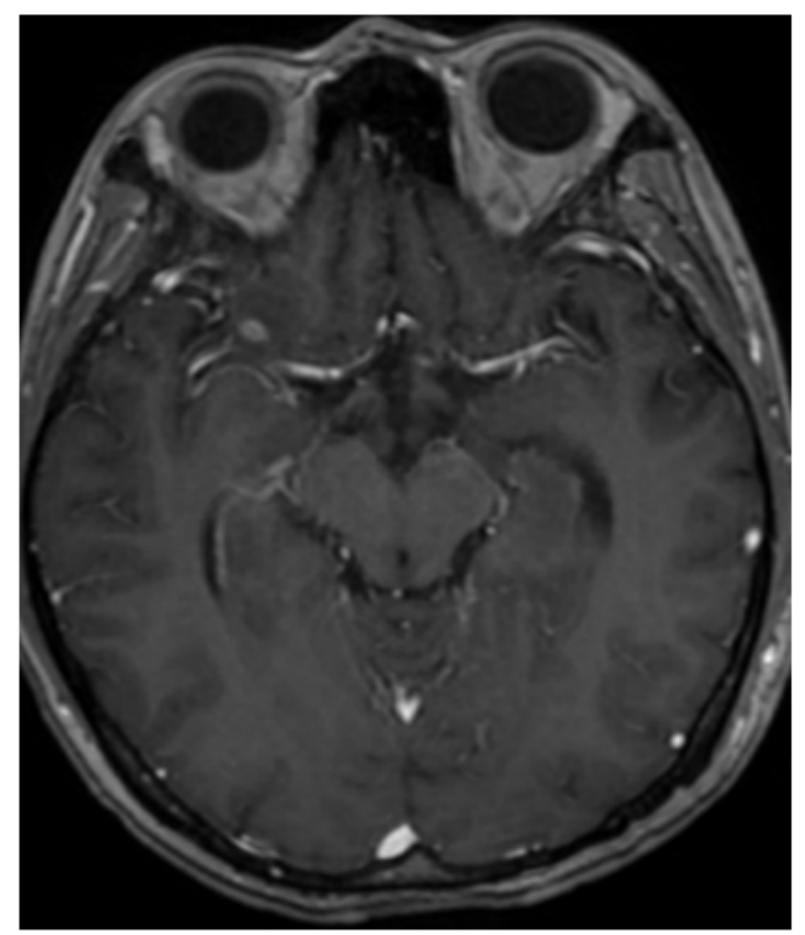

$\mathrm{B}$

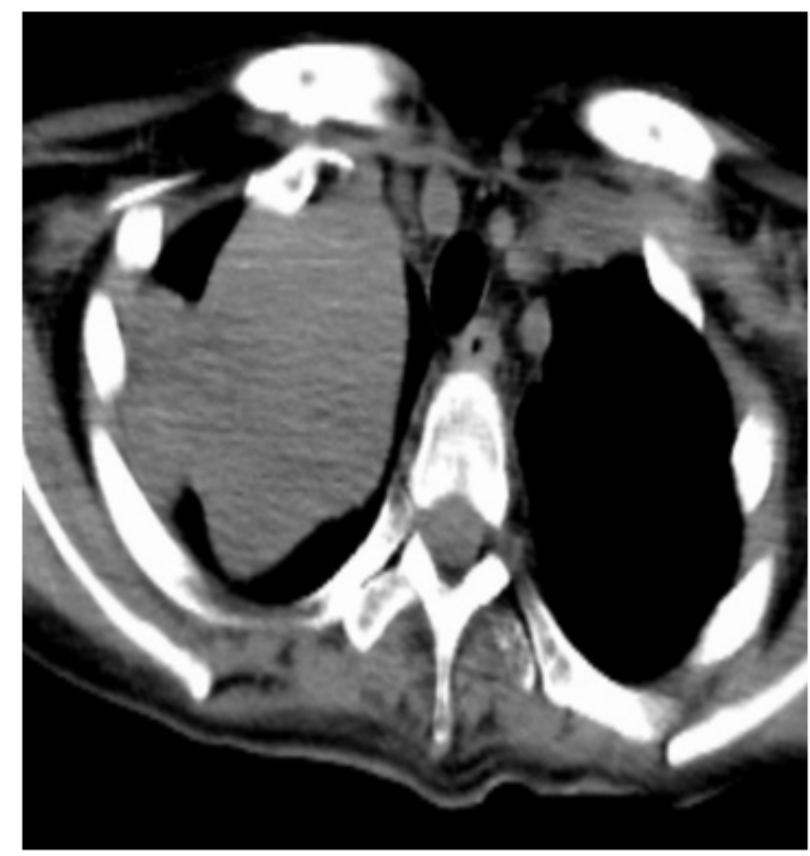

D

\section{Figure 6}

Imaging two months after radiotherapy: Magnetic resonance imaging (A) showing tumor in the head and neck were still reduced. Magnetic resonance imaging ( $B$ and $C$ ) depicted multiple intracranial metastases. Computed tomography scans (D) revealed increased enlargement of right lung lesions and were larger than prior assessment. 